\title{
Treatment Failure of Isavuconazole in a Patient with Cryptococcosis
}

\author{
Kathleen A. Linder • Tejal N. Gandhi • Marisa H. Miceli $\mathbb{D}$
}

Received: 27 June 2019/Accepted: 16 August 2019/Published online: 26 August 2019

(C) Springer Nature B.V. 2019

\begin{abstract}
Isavuconazole is a broad-spectrum azole that is FDA-approved for the treatment of aspergillosis and mucormycosis; data on the use of isavuconazole for the treatment and prevention of other invasive fungal infections are limited. Here, we report a patient with pulmonary cryptococcosis treated with isavuconazole who experienced progression to disseminated infection with Cryptococcus while on isavuconazole. Caution is advised when using isavuconazole in situations where there is a paucity of data to recommend its use.
\end{abstract}

Keywords Cryptococcus · Isavuconazole · Treatment failure

\section{Introduction}

Isavuconazole is an extended-spectrum azole with broad activity against yeasts, molds, and dimorphic

Handling Editor: Vishnu Chaturvedi.

K. A. Linder · T. N. Gandhi · M. H. Miceli ( $₫)$

Division of Infectious Diseases, University of Michigan

Medical School, 1500 E. Medical Dr., South University

Hospital F4005, Ann Arbor, MI 48109-5378, USA

e-mail: mmiceli@med.umich.edu

K. A. Linder

Veterans Affairs Ann Arbor Healthcare System,

Ann Arbor, MI, USA fungi [1]. It was approved by the FDA in 2015 for the treatment of invasive aspergillosis and mucormycosis, but has been used off-label for the treatment of other fungal infections [1, 2]. Despite a lack of randomized controlled trials on the use of isavuconazole for other fungal species, favorable pharmacokinetics and lowside-effect profile have led to the use of isavuconazole as an alternative for both treatment and prophylaxis of many invasive fungal infections [1, 3, 4]. Herein, we describe a case of cryptococcal pneumonia that progressed to disseminated infection in a patient receiving isavuconazole.

\section{Case}

A 60-year-old man with a history of type 2 diabetes and factor $\mathrm{V}$ Leiden heterozygosity was transferred to our hospital for the management of ventilator-dependent respiratory failure and acute respiratory distress syndrome secondary to influenza A pneumonia.

While in the intensive care unit, he developed pulmonary embolism requiring thrombolysis, tension pneumothorax that was decompressed by several chest tubes, and ventilator-associated pneumonia with multi-drug-resistant Acinetobacter baumannii complex.

On hospital day 47, the patient had a bronchoscopy with bronchoalveolar lavage (BAL) due to ongoing respiratory failure and worsening leukocytosis despite appropriate antibiotic treatment for bacterial 
pneumonia. BAL culture yielded Cryptococcus neoformans. A subsequent computed tomography scan showed diffuse ground glass opacities, patchy areas of consolidation throughout both lungs, and a previously described complex, septated pneumothorax with chest tubes in place. Because of the presence of C. neoformans in the lungs, a lumbar puncture was performed. This study was negative for nucleated cells; protein was $72 \mathrm{mg} / \mathrm{dl}$ and glucose $97 \mathrm{mg} / \mathrm{dl}$. Serum and cerebrospinal fluid cryptococcal antigen were negative. Fungal blood cultures were obtained and were negative. The patient was started on oral fluconazole $400 \mathrm{mg}$ daily for the treatment of cryptococcal pneumonia. The treating team was made aware of potential drug-drug interactions given the concurrent use of fluconazole and amiodarone.

After 8 days of fluconazole therapy (hospital day 58), the patient's corrected QT interval on electrocardiogram increased from 445 to $544 \mathrm{~ms}$. Fluconazole was discontinued, and intravenous isavuconazole was initiated at a loading dose of $372 \mathrm{mg}$ every $8 \mathrm{~h}$ for 6 doses and then $372 \mathrm{mg}$ daily.

Six days after initiating isavuconazole (hospital day 64), the patient developed persistent fevers to $39.4^{\circ} \mathrm{C}$ and leukocytosis to 12,800 cells $/ \mu$ l. This was initially thought to represent relapsing ventilator-associated pneumonia, and the patient's antibiotics were broadened to include vancomycin and both inhaled colistin and intravenous colistin. Blood cultures were collected; 1 of 2 sets was positive for C. neoformans, despite ongoing treatment with isavuconazole. Given the progression to disseminated cryptococcosis on isavuconazole, antifungal treatment was switched to intravenous liposomal amphotericin B at a dose of $3 \mathrm{mg} / \mathrm{kg}$ daily. Repeat blood cultures demonstrated the clearance of fungemia. He remained on liposomal amphotericin B for 3 weeks, while other QT-prolonging medications were gradually discontinued; at that point, he was able to complete treatment with fluconazole and had no further recurrence of cryptococcal disease.

Cryptococcus neoformans antifungal susceptibilities were performed at the University of Texas Health Sciences Center and demonstrated a fluconazole minimum inhibitory concentration (MIC) of $2 \mathrm{mcg} /$ $\mathrm{ml}$, an amphotericin B MIC of $0.5 \mathrm{mcg} / \mathrm{ml}$, and an isavuconazole breakpoint of $<0.03 \mathrm{mcg} / \mathrm{ml}$. Serum isavuconazole trough level was $2.1 \mathrm{mcg} / \mathrm{ml}$ (Viracor Eurofins, Lee's Summit, MO).

\section{Discussion}

In the case above, we discuss a case of cryptococcal pneumonia that progressed to disseminated cryptococcosis despite ongoing treatment with isavuconazole. While isavuconazole is approved for the treatment of invasive aspergillosis and mucormycosis, its favorable safety profile has led to widespread adoption of this agent as an alternative for the treatment of other fungal infections, as well as for prophylaxis in high-risk patients [1]. For example, our patient received isavuconazole because he was unable to receive the recommended agent (fluconazole) due to a prolonged corrected QT interval. However, the clinical efficacy of isavuconazole for non-FDA-approved indications remains unknown [1,2]. As the use of isavuconazole continues to expand, more information from the clinical setting has become available. There is growing evidence to support that breakthrough invasive mold infections on isavuconazole are an emerging problem, but failures of isavuconazole for the treatment of Cryptococcus have not been previously reported $[5,6]$.

Isavuconazole has been shown to have in vitro activity against both $C$. neoformans and $C$. gattii, with MIC ranging between 0.008 and $0.5 \mu \mathrm{g} / \mathrm{ml}$ across species [7-9]. One multicenter study suggests an epidemiologic cutoff value (ECV; the upper limit MIC for a wild type isolate) of $0.06-0.25 \mu \mathrm{g} / \mathrm{ml}$ for the $C$. neoformans-C. gattii complex [9]. There is currently no standardized clinical breakpoint to predict clinical success or failure with the use of isavuconazole for the treatment of Cryptococcus [10]. In our case, the Cryptococcus isolate was presumably sensitive to isavuconazole as the MIC was below the measured breakpoint (MIC < 0.03).

The mechanism behind this patient's treatment failure is unclear, particularly in the setting of infection with an isolate with low MIC. The global SENTRY antifungal surveillance program showed that all 84 C. neoformans clinical isolates had an MIC below the breakpoint to fluconazole, itraconazole, voriconazole, and posaconazole, although correlation between MIC and treatment outcome was not studied; at that time, isavuconazole was not available [11]. In recent years, Cryptococcus isolates with increased azole MICs (including to isavuconazole) have emerged [7, 12]. Whether a low MIC correlates with treatment response is unknown. A recent small study 
demonstrated that fluconazole MICs of either $<16$ $\mu \mathrm{g} / \mathrm{ml}$ versus $\geq 16 \mu \mathrm{g} / \mathrm{ml}$ did not correlate with clinical success or failure, respectively, and that the only accurate predictor of poor outcome was disseminated disease [12]. Perhaps a similar discordance between in vitro and in vivo data exists with isavuconazole, such that a sensitive MIC is unable to accurately predict clinical outcome.

Studies of breakthrough candidemia have described a high prevalence of breakthrough infection with sensitive isolates [13]. In one study of patients with hematologic malignancies, all seven cases of candidemia that broke through fluconazole or posaconazole maintained sensitivity to the agent used for prophylaxis [13]. These authors suggest that host factors, including hypogammaglobulinemia and overall immunosuppression, may be more important than antifungal prophylaxis in this patient population [13]. However, though our patient may be considered at risk for IFI from his ICU admission and his recent influenza infection, he did not have significant risk factors such as profound neutropenia or hematologic malignancy. Additionally, while many of these Candida infections were attributed to biofilms from central venous catheter placement, the pathophysiology of cryptococcosis differs.

Linear pharmacokinetics and high bioavailability are distinctive characteristics of isavuconazole [1]. These features contrast with the narrow therapeutic index, nonlinear kinetics and high intra-individual variability of posaconazole and voriconazole that make therapeutic drug monitoring (TDM) an important tool for the management of patients receiving these drugs [14]. Several studies have shown increasing isavuconazole trough levels over time during treatment. Our patient's serum isavuconazole trough level was $2.1 \mathrm{mcg} / \mathrm{ml}$, which is within the therapeutic range of $2-4 \mathrm{mg} / \mathrm{L}$ that has been proposed by some authors [15]. However, no clear concentration thresholds have been identified for efficacy, and TDM of isavuconazole is not currently recommended [16, 17].

Regardless of the mechanism of our patient's treatment failure, real-world data using isavuconazole for the treatment of cryptococcosis are lacking. Thompson and colleagues reviewed a subset of nine patients from the VITAL study with proven cryptococcosis; of these patients, six had a successful response to isavuconazole and two had stable disease after a course of therapy [2]. Only one patient expired during treatment with isavuconazole, and death was attributed to concurrent disseminated nocardiosis [2]. The small sample size of this study limits broad conclusions regarding the efficacy of isavuconazole for the treatment of cryptococcosis. As such, isavuconazole is not currently recommended for primary therapy of cryptococcosis due to limited evidence [18].

In conclusion, the role of isavuconazole in the treatment of cryptococcosis is limited. Caution is recommended when using isavuconazole for off-label indications. Further and larger studies are warranted.

\section{Compliance with Ethical Standards}

Ethical Statement The authors confirm that this material is original and has not been published in whole or in part elsewhere; that the manuscript is not currently being considered for publication in another journal; and that all authors have been personally and actively involved in substantive work leading to the manuscript, and will hold themselves jointly and individually responsible for its content. In addition, the authors confirm that they have completed the checklist for submission of a case report to Mycopathologia published by Bouchara et al. [19].

\section{References}

1. Miceli MH, Kauffman CA. Isavuconazole: a new broadspectrum triazole antifungal agent. Clin Infect Dis. 2015;61(10):1558-65.

2. Thompson GR 3rd, Rendon A, Dos Santos RR, QueirozTelles F, Ostrosky-Zeichner L, Azie N, et al. Isavuconazole treatment of cryptococcosis and dimorphic mycoses. Clin Infect Dis. 2016;63(3):356-62.

3. Keirns J, Desai A, Kowalski D, Lademacher C, Mujais S, Parker B, et al. QT interval shortening with isavuconazole: in vitro and in vivo effects on cardiac repolarization. Clin Pharmacol Ther. 2017;101(6):782-90.

4. Mellinghoff SC, Bassetti M, Dorfel D, Hagel S, Lehners N, Pils A, et al. Isavuconazole shortens the QTc interval. Mycoses. 2018;61(4):256-60.

5. Fung M, Schwatrz BS, Doernberg SB, Langelier C, Lo M, Graff L, et al. Breakthrough invasive fungal infections on isavuconazole prophylaxis and treatment: what is happening in the real-world setting? Clin Infect Dis. 2018;67:1142-3.

6. Rausch CR, DiPippo AJ, Bose P, Kontoyiannis DP. Breakthrough fungal infections in leukemia patients receiving isavuconazole. Clin Infect Dis. 2018;67(10):1610-3.

7. Datta K, Rhee P, Byrnes E 3rd, Garcia-Effron G, Perlin DS, Staab JF, et al. Isavuconazole activity against Aspergillus lentulus, Neosartorya udagawae, and Cryptococcus gattii, emerging fungal pathogens with reduced azole susceptibility. J Clin Microbiol. 2013;51(9):3090-3. 
8. Pfaller MA, Rhomberg PR, Wiederhold NP, Gibas C, Sanders $\mathrm{C}$, Fan $\mathrm{H}$, et al. In vitro activity of isavuconazole against opportunistic pathogens from two mycology reference laboratories. Antimicrob Agent Chemother. 2018;62(10):e01230-18.

9. Espinel-Ingroff A, Chowdhary A, Gonzalez GM, Guinea J, Hagen F, Meis JF, et al. Multicenter study of isavuconazole MIC distributions and epidemiological cutoff values for the Cryptococcus neoformans-Cryptococcus gattii species complex using the CLSI M27-A3 broth microdilution method. Antimicrob Agent Chemother. 2015;59(1):666-8.

10. CLSI subcommittee on antifungal susceptibility. Meeting resources, January 2018. Accessed https://clsi.org/ meetings/sub-antifungal/ on 14 Mar 2019.

11. Castanhiera M, Messer SA, Rhomberg PR, Pfaller MA. Antifungal susceptibility patterns of a global collection of fungal isolates: results of the SENTRY Antifungal Surveillance Program (2013). Diagn Microbiol Infect Dis. 2016;85:200-4.

12. Vena A, Munoz P, Guinea J, Escribano P, Pelaez T, Valerio $\mathrm{M}$, et al. Fluconazole resistance is not a predictor of poor outcome in patients with cryptococcosis. Mycoses. 2019;62:441-9.

13. Gamaletsou MN, Daikos GL, Walsh TJ, Perlin DS, Ortigosa CJ, Psaroulaki A, et al. Breakthrough candidaemia caused by phenologically susceptible Candida spp in patients with haematological malignancies does not correlate with established interpretive breakpoints. Int $\mathbf{J}$ Antimicrob Agent. 2014;44:248-55.

14. Patterson TF, Thompson GR 3rd, Denning DW, Fishman JA, Hadley S, Herbrecht R, et al. Practice guidelines for the diagnosis and management of aspergillosis: 2016 update by the Infections Diseases Society of America. Clin Infect Dis. 2016;63:e1-60.

15. Committee SIFIG. Dutch working party on antibiotic therapy. SWAB guidelines for the management of invasive fungal infections. Revised version. Released: 14 Dec 2017. http://www.swab.nl/swab/cms3.nsf/uploads/3AA7A56 CE879587BC12581F80061297F/\$FILE/SWAB\% 20Richtlijn\%20Mycosen\%202017\%20(final).pdf.

16. Kim P, Tegtmeier B, Dadwal S, Ito J, Kriengkauykiat J. Correlating isavuconazole serum levels with efficacy and adverse effects (AE) among immunocompromised patients. Open Forum Infect Dis. 2017;4:S293.

17. Furfaro E, Signori A, Di Grazia C, Dominietto A, Raiola AM, Aquino S, Ghiggi C, Ghiso A, Ungaro R, Angellucci E, Viscoli C, Mikulska M. Serial monitoring of isavuconazole blood levels during prolonged antifungal therapy. J Antimicrob Chemother 2019;74(8):2341-2346.

18. Perfect JR, Dismukes WE, Dromer F, Goldman DL, Graybill JR, Hamill RJ, et al. Clinical practice guidelines for the treatment of cryptococcal disease: 2010 update by the Infectious Diseases Society of America. Clin Infect Dis. 2010;50(3):291-322.

19. Bouchara JP, Chaturvedi V. The curious case of "case report" of infections caused by human and animal fungal pathogens: an educational tool, an online archive, or a format in need of retooling. Mycopathologia. 2018;183(6):879-91.

Publisher's Note Springer Nature remains neutral with regard to jurisdictional claims in published maps and institutional affiliations. 